\title{
ON THE CARDINALITY OF ALMOST DISCRETELY LINDELÖF SPACES
}

\author{
ANGELO BELLA AND SANTI SPADARO
}

\begin{abstract}
A space is said to be almost discretely Lindelöf if every discrete subset can be covered by a Lindelöf subspace. In [10, Juhász, Tkachuk and Wilson asked whether every almost discretely Lindelöf first-countable Hausdorff space has cardinality at most continuum. We prove that this is the case under $2^{<\mathfrak{c}}=\mathfrak{c}$ (which is a consequence of Martin's Axiom, for example) and for Urysohn spaces in ZFC, thus improving a result by Juhász, Soukup and Szentmiklóssy from [9]. We conclude with a few related results and questions.
\end{abstract}

\section{INTRODUCTION}

In [1] Arhangel'skii published his celebrated theorem stating that every Lindelöf first-countable Hausdorff space has cardinality at most continuum. Besides solving a long standing question due to Alexandroff and Urysohn, Arhangel'skii's Theorem gave a definite boost to the area of cardinal invariants in topology, inspiring new techniques, results and questions that continue to be the object of current research (see [7] for a survey on Arhangel'skii's Theorem and its legacy).

Recall that a space is said to be discretely Lindelöf if the closure of every discrete set is Lindelöf. A well-known question due to Arhangel'skii asks whether every regular discretely Lindelöf space is Lindelöf.

A space $X$ is defined to be almost discretely Lindelöf [10] if for every discrete set $D \subset X$ there is a Lindelöf subspace $L$ of $X$ such that $D \subset L$. Of course every discretely Lindelöf space is almost discretely Lindelöf. Any example of an $S$-space (a regular hereditarily separable non-Lindelöf space) provides an (alas, only consistent) example of an almost discretely Lindelöf non-Lindelöf regular space. It is still open whether there exists an example of such a space in ZFC.

2000 Mathematics Subject Classification. Primary: 54A25, 54D20; Secondary: 54D35, 54D10, 54D55.

Key words and phrases. Cardinal inequality, Lindelöf space, Arhangel'skii Theorem, elementary submodel, left-separated set, right-separated set, discrete set, free sequence. 
The authors of [10] prove that every almost discretely Lindelöf firstcountable space has cardinality at most $2^{\mathfrak{c}}$ and ask whether every almost discretely Lindelöf first-countable Hausdorff space has cardinality bounded by the continuum. We prove that this is the case for Urysohn spaces and for Hausdorff spaces under $2^{<\mathfrak{c}}=\mathfrak{c}$. Actually we prove a little more than that, namely: 1) every almost discretely Lindelöf sequential space such that $\psi(X) \leq \mathfrak{c}$ has cardinality at most continuum and 2) every almost discretely Lindelöf Hausdorff space such that $\psi_{c}(X) \cdot t(X)=\omega$ has cardinality at most continuum.

We should mention that in [9] Juhász, Soukup and Szentmiklóssy proved that every almost discretely Lindelöf first-countable regular space has cardinality bounded by the continuum.

We conclude by exploring a few further generalizations and related results.

In our proofs we will often use elementary submodels of the structure $(H(\mu), \epsilon)$. Dow's survey 5 is enough to read our paper, and we give a brief informal refresher here. Recall that $H(\mu)$ is the set of all sets whose transitive closure has cardinality smaller than $\mu$. When $\mu$ is regular uncountable, $H(\mu)$ is known to satisfy all axioms of set theory, except the power set axiom. We say, informally, that a formula is satisfied by a set $S$ if it is true when all bounded quantifiers are restricted to $S$. A set $M \subset H(\mu)$ is said to be an elementary submodel of $H(\mu)$ (and we write $M \prec H(\mu)$ ) if a formula with parameters in $M$ is satisfied by $H(\mu)$ if and only if it is satisfied by $M$.

The downward Löwenheim-Skolem theorem guarantees that for every $S \subset H(\mu)$, there is an elementary submodel $M \prec H(\mu)$ such that $|M| \leq|S| \cdot \omega$ and $S \subset M$. This theorem is enough in many applications, but it is often useful (especially in cardinal bounds for topological spaces) to have the following closure property. We say that $M$ is $\kappa$ closed if for every $S \subset M$ such that $|S| \leq \kappa$ we have $S \in M$. For every countable set $S \subset H(\mu)$ there is always a $\kappa$-closed elementary submodel $M \prec H(\mu)$ such that $|M|=2^{\kappa}$ and $S \subset M$.

The following theorem is also used often: let $M \prec H(\mu)$ such that $\kappa+1 \subset M$ and $S \in M$ be such that $|S| \leq \kappa$. Then $S \subset M$.

All spaces under consideration are assumed to be $T_{1}$. Undefined notions can be found in [6] for topology and [11] for set theory. Our notation regarding cardinal functions mostly follows [8]. In particular, $\psi(X)$ and $t(X)$, denote the pseudocharacter and tightness of $X$ respectively. We recall the definition of these two important cardinal functions, given that they are essential for many of the results in our paper. 
Let $A$ be a subset of $X$. The pseudocharacter of $A$ in $X(\psi(A, X))$ is defined as the minimum cardinal $\kappa$ such that $A$ is the intersection of $\kappa$ many open sets. We denote $\psi(\{x\}, X)$ by $\psi(x, X)$. The pseudocharacter of the space $X$ is defined as $\psi(X)=\sup \{\psi(x, X): x \in X\}$.

The tightness of the point $x$ in the space $X(t(x, X))$ is defined as the minimum cardinal $\kappa$ such that for every set $A \subset X$ such that $x \in \bar{A} \backslash A$ there is a $\leq \kappa$-sized set $B \subset A$ such that $x \in \bar{B}$. The tightness of the space $X$ is defined as $t(X)=\sup \{t(x, X): x \in X\}$.

\section{The MAIN RESUlts}

Recall that a space is right-separated if and only if it admits a wellordering where every initial segment is open. It is well-known and easy to prove that a space is right-separated if and only if it is scattered (that is, every non-empty subset contains an isolated point). We denote by $h(X)$ the supremum of the cardinalities of the right-separated subsets of $X$. As is well known (see, for example, [8], 2.9), $h L(X)=h(X)$ for every space $X$, where $h L(X)$ denotes the hereditarily Lindelöf degree of $X$.

We denote by $g(X)$ the supremum of the cardinalities of the closures of discrete sets in $X$. Since every scattered space has a dense discrete subset we have $h(X) \leq g(X)$.

We start with an observation contained in the proof of Theorem 4 from [9] which we would like to isolate for the convenience of the reader.

Lemma 1. 9] Let $X$ be an almost discretely Lindelöf $T_{2}$ space $X$. Then $h L(X) \leq 2^{\chi(X)}$.

Proof. Recall (see for example 2.5 of [8] ) that $|Y| \leq d(Y)^{\chi(Y)}$ for every Hausdorff space $Y$ and hence $|\bar{D}| \leq|D|^{\chi(X)}$ for every $D \subset X$. But since $X$ is almost discretely Lindelöf, for every discrete set $D \subset X$, there is a Lindelöf space $L \subset X$ such that $D \subset L$. It follows that $|\bar{D}| \leq 2^{\chi(X)}$, by Arhangel'skii's Theorem. Taking suprema we obtain that $g(X) \leq 2^{\chi(X)}$. Now $h L(X) \leq g(X)$.

Definition 2. [12] For any space $X$ and any set $A \subseteq X, C_{\theta}(A)$ is the set of all points $x$ such that $\bar{U} \cap A \neq \emptyset$ for every neighbourhood $U$ of $x$. $A$ is $\theta$-closed if $A=C l_{\theta}(A)$. The $\theta$-closure $[A]_{\theta}$ is the smallest $\theta$-closed set containing $A$.

Lemma 3. Let $X$ be an almost discretely Lindelöf Hausdorff space, and $A$ be a $\theta$-closed set. Then $\psi(A, X) \leq 2^{\chi(X)}$, that is $A$ is the intersection of a family of $2^{\chi(X)}$ open sets. 
Proof. For each $x \in X \backslash A$ we may fix an open neighbourhood $U_{x}$ of $x$ such that $\overline{U_{x}} \cap A=\emptyset$. By Lemma 1, there is a set $S \subseteq X \backslash A$ such that $|S| \leq 2^{\chi(X)}$ and $\bigcup\left\{U_{x}: x \in S\right\}=X \backslash A$. Then $A=\bigcap\left\{X \backslash \overline{U_{x}}\right.$ : $x \in S\}$.

Definition 4. We say that a sequence $\left\{x_{\alpha}: \alpha<\kappa\right\}$ is $\theta$-free if:

$$
\left[\left\{x_{\alpha}: \alpha<\beta\right\}\right]_{\theta} \cap \overline{\left\{x_{\alpha}: \beta \leq \alpha<\kappa\right\}}=\emptyset
$$

for every $\beta<\kappa$. The cardinal function $F_{\theta}(X)$ denotes the supremum of the cardinalities of all $\theta$-free sequences contained in $X$.

Recall that a sequence $\left\{x_{\alpha}: \alpha<\kappa\right\}$ is called free if

$$
\overline{\left\{x_{\alpha}: \alpha<\beta\right\}} \cap \overline{\left\{x_{\alpha}: \beta \leq \alpha<\kappa\right\}}=\emptyset
$$

for every $\beta<\kappa$. The cardinal function $F(X)$ is defined as the supremum of the cardinalities of free sequences contained in $X$. Free sequences are an important tool in Arhangel'skii's original solution of the Alexandroff-Urysohn problem.

Obviously, every $\theta$-free sequence is free, so $F_{\theta}(X) \leq F(X)$ and $F(X)=F_{\theta}(X)$ for every regular space $X$. However, the inequality may be strict for non-regular spaces (see Section 3 ).

The following lemma is proved via a simple standard argument.

Lemma 5. In an almost discretely Lindelöf space $X$, every free sequence has length at most $\chi(X)$.

Recall that the closed pseudocharacter of the point $x$ in the space $X\left(\psi_{c}(x, X)\right)$ is defined as the minimum cardinal $\kappa$ such that there is a family $\left\{U_{\alpha}: \alpha<\kappa\right\}$ of neighbourhoods of $x$ such that $\bigcap\left\{\overline{U_{\alpha}}: \alpha<\right.$ $\kappa\}=\{x\}$. The closed pseudocharacter of the space $X$ is then defined as $\psi_{c}(X)=\sup \left\{\psi_{c}(x, X): x \in X\right\}$. It is easy to see that $\psi_{c}(X) \leq \chi(X)$, for every Hausdorff space $X$.

Recall that a space $X$ is Urysohn if for every pair of distinct points $x, y \in X$ there are open neighbourhoods $U$ of $x$ and $V$ of $y$ such that $\bar{U} \cap \bar{V}=\emptyset$.

The proof of the following theorem is a variation on the proof of Theorem 2.3 from [2].

Theorem 6. Let $X$ be a space such that $F_{\theta}(X) \leq \kappa$ and $\psi\left([F]_{\theta}, X\right) \leq$ $2^{\kappa}$, for every $\theta$-free sequence $F \subset X$. Then there exists $A \subset X$ such that $|A| \leq 2^{\kappa}$ and $X=[A]_{\theta}$.

Proof. Let $\mu$ be a large enough regular cardinal and $M$ be a $\kappa$-closed elementary submodel of $H(\mu)$ such that $X \in M,|M|=2^{\kappa}$ and $2^{\kappa}+1 \subset$ $M$. 
Claim 1. Let $F$ be a $\theta$-free sequence in $X$ contained in $X \cap M$ and let $p$ be a point outside of $[F]_{\theta}$. Then there is an open set $U \in M$ such that $[F]_{\theta} \subset U$ and $p \notin U$.

Proof of Claim 1. Since $F$ is a $\theta$-free sequence in $X$, the cardinality of $F$ does not exceed $\kappa$, so $F \in M$. Hence, by elementarity, we also have that $[F]_{\theta} \in M$. Now $\psi\left([F]_{\theta}, X\right) \leq 2^{\kappa}$, so we can fix an open family $\mathcal{U} \in M$ having cardinality $2^{\kappa}$, such that $[F]_{\theta}=\bigcap \mathcal{U}$. Note that $\mathcal{U} \subset M$. Now pick $U \in \mathcal{U}$ such that $p \notin U$ and note that $U$ satisfies the requirement of Claim 1.

Claim 2. $X=[X \cap M]_{\theta}$.

Proof of Claim 2. Suppose that the statement of the claim is false and pick a point $x \notin[X \cap M]_{\theta}$. Then we can inductively find points $\left\{x_{\alpha}\right.$ : $\left.\alpha<\kappa^{+}\right\} \subset X \cap M$ and open sets $\left\{U_{\alpha}: \alpha<\kappa^{+}\right\} \subset M$ such that:

(1) $\left[\left\{x_{\alpha}: \alpha<\beta\right\}\right]_{\theta} \subset U_{\beta}$.

(2) $x \notin U_{\beta}$.

(3) $x_{\beta} \notin \bigcup\left\{U_{\alpha}: \alpha \leq \beta\right\}$, for every $\beta<\kappa^{+}$.

To see that, suppose for a given $\delta<\kappa^{+}$we have constructed $\left\{\left(x_{\alpha}, U_{\alpha}\right)\right.$ : $\alpha<\delta\}$ satisfying the three conditions above up to $\delta$. Note that $\left\{x_{\alpha}: \alpha<\delta\right\}$ is a $\theta$-free sequence. Indeed, from the first and third condition it follows that $\left[\left\{x_{\alpha}: \alpha<\gamma\right\}\right]_{\theta} \subset U_{\gamma}$ and $\left\{x_{\alpha}: \gamma \leq \alpha<\delta\right\} \subset X \backslash U_{\gamma}$, which implies that $\left[\left\{x_{\alpha}: \alpha<\gamma\right\}\right]_{\theta} \cap \overline{\left\{x_{\alpha}: \gamma \leq \alpha<\delta\right\}}=\emptyset$.

Therefore, we can use Claim 1 to find an open set $U_{\delta}$ such that $\left[\left\{x_{\alpha}: \alpha<\delta\right\}\right]_{\theta} \subset U_{\delta}$ and $x \notin U_{\delta}$.

By elementarity, we are now allowed to pick a point $x_{\delta} \in X \cap M \backslash$ $\bigcup\left\{U_{\alpha}: \alpha \leq \delta\right\}$ and thus continue the induction.

It is easy to see that eventually $\left\{x_{\alpha}: \alpha<\kappa^{+}\right\}$is a $\theta$-free sequence in $X$ of cardinality $\kappa^{+}$, which contradicts $F_{\theta}(X) \leq \kappa$.

Since in a Urysohn space $X$ the inequality $\left|[A]_{\theta}\right| \leq|A|^{\chi(X)}$ holds for any set $A \subset X$ (see [4]), we immediately get:

Corollary 7. If $X$ is a Urysohn almost discretely Lindelöf space, then $|X| \leq 2^{\chi(X)}$.

Recall that a space is sequential if every non-closed set contains a sequence converging outside of it. It is easy to see that a closure of a subspace in a sequential space is obtained by iterating the sequential closure at most $\omega_{1}$ many times. Every first-countable space is sequential and every sequential space has countable tightness. 
Theorem 8. $\left(2^{<\mathfrak{c}}=\mathfrak{c}\right)$. Let $X$ be a $T_{2}$ sequential almost discretely Lindelöf space such that $\psi(X) \leq \mathfrak{c}$. Then $|X| \leq \mathfrak{c}$.

Proof. Let $\mu$ be a large enough regular cardinal. Let $M$ be a $<\mathfrak{c}$ closed elementary submodel of $H(\mu)$ such that $|M|=\mathfrak{c}, \mathfrak{c}+1 \subset M$ and $X \in M$.

From the fact that $X$ is a Hausdorff sequential space and the fact that $M$ is $\omega$-closed it follows that $X \cap M$ is a closed subset of $X$.

We claim that $d(X) \leq \mathfrak{c}$, and that would finish the proof, because every sequential space of density continuum has cardinality continuum.

Suppose by contradiction that $d(X) \geq \mathfrak{c}^{+}$. Using that, it is easy to find a left-separated subset $L$ of $X$ having cardinality $\mathfrak{c}^{+}$. Without loss we can assume that $L \in M$.

Since $L$ has cardinality larger than the continuum, we can pick a point $p \in L \backslash M$. Fix a point $x \in X \cap M$. Then we can find a family $\mathcal{U}_{x} \in M$ of cardinality continuum such that $\bigcap \mathcal{U}_{x}=\{x\}$. Since $\left|\mathcal{U}_{x}\right| \leq \mathfrak{c}$ and $\mathfrak{c}+1 \subset M$ we actually have that $\mathcal{U}_{x} \subset M$. Hence, for every $x \in X \cap M$, we can find an open set $U_{x} \in M$ such that $x \in U_{x}$ and $p \notin U_{x}$.

Now $\mathcal{U}=\left\{U_{x}: x \in X \cap M\right\}$ is an open cover of $X \cap M$.

Claim. There is a $<\mathfrak{c}$-sized subcollection of $\mathcal{U}$ covering $L \cap M$.

Proof of Claim. If $L \cap M$ had cardinality smaller than the continuum, this would be trivially true. So we can assume that $|L \cap M|=\mathfrak{c}$.

Let $\left\{U_{\alpha}: \alpha<\mathfrak{c}\right\}$ be an enumeration of $\mathcal{U}$ in type $\mathfrak{c}$ and set $V_{\alpha}=$ $U_{\alpha} \backslash \bigcup\left\{U_{\beta}: \beta<\alpha\right\}$. Suppose by contradiction that the statement of the Claim is not true. Then the set $S=\left\{\alpha<\mathfrak{c}: V_{\alpha} \cap(L \cap M) \neq \emptyset\right\}$ has cardinality continuum.

Pick a point $x_{\alpha} \in V_{\alpha} \cap L$, for every $\alpha \in S$. Then $R=\left\{x_{\alpha}: \alpha \in S\right\}$ is a set of size continuum which is both right-separated and left-separated. So by 2.12 of [8] the set $R$ contains a discrete set $D$ having cardinality continuum.

Since $X$ is almost discretely Lindelöf, we can find a Lindelöf subspace $Y \subset X$. such that $D \subset Y$. Now, $X \cap M$ being closed, the set $Y \cap M$ is also Lindelöf, and since $\mathcal{U}$ covers $Y \cap M$, we can find an ordinal $\delta<\mathfrak{c}$ such that $D \subset Y \cap M \subset \bigcup\left\{U_{\alpha}: \alpha<\delta\right\}$. But since $D$ has cardinality continuum, there must be $\gamma>\delta$ such that $D \cap V_{\gamma} \neq \emptyset$ and this contradicts the fact that $V_{\gamma}$ is disjoint from $\bigcup\left\{U_{\alpha}: \alpha<\delta\right\}$.

Fix a subcollection $\mathcal{V} \subset \mathcal{U}$ of cardinality smaller than the continuum such that $L \cap M \subset \cup \mathcal{V}$.

Since $M$ is $<\mathfrak{c}$-closed we have that $\mathcal{V} \in M$ and since $L$ is also an element of $M$ it follows that: $M \models L \subset \cup \mathcal{V}$. 
By elementarity $H(\mu) \models L \subset \bigcup \mathcal{V}$, but that is a contradiction because $p \in L \backslash \bigcup \mathcal{V}$.

Theorem 9. $\left(2^{<\mathfrak{c}}=\mathfrak{c}\right)$ Let $X$ be an almost discretely Lindelöf Hausdorff space such that $\psi_{c}(X) \cdot t(X)=\omega$. Then $|X| \leq \mathfrak{c}$.

Proof. Let $M$ be given as in the proof of Theorem 8 . The proof is essentially the same as the proof of Theorem 8 , except that the argument proving that $X \cap M$ is closed is different. Here it is. Let $x \in \overline{X \cap M}$. Pick a family $\left\{U_{n}: n<\omega\right\}$ of neighbourhoods of $x$ such that $\bigcap\left\{\overline{U_{n}}: n<\omega\right\}=\{x\}$. Use the fact that the tightness of $X$ is countable to pick a countable set $C \subset X \cap M$ such that $x \in \bar{C}$. Note that, since $M$ is $\omega$-closed the set $U_{n} \cap C$ belongs to $M$, for every $n<\omega$. From elementarity and $\omega$-closedness of $M$ again it follows that $\{x\}=\bigcap\left\{\overline{U_{n} \cap C}: n<\omega\right\}$ is also an element of $M$. Hence $x \in X \cap M$ and this concludes the proof that $X \cap M$ is closed.

Question 1. Are Theorems 8 and 9 true in ZFC?

\section{ODDS AND ENDS}

A cellular family is a family of pairwise disjoint non-empty open sets. The cellularity of $X(c(X))$ is defined as the supremum of the cardinalities of the cellular families in $X$.

The following is a natural generalization of the notion of an almost discretely Lindelöf space.

Definition 10. We define a space $X$ to be cellular-Lindelöf if for every cellular family $\mathcal{U}$ there is a Lindelöf subspace $L \subset X$ such that $U \cap L \neq$ $\emptyset$, for every $U \in \mathcal{U}$.

The following proposition follows immediately from the definition

\section{Proposition 11.}

(1) Every ccc space is cellular-Lindelöf.

(2) Every Lindelöf space is cellular-Lindelöf.

(3) Every almost discretely Lindelöf space is cellular-Lindelöf.

So the cellular-Lindelöf property turns out to be a common weakening of the countable chain condition and the Lindelöf property, in a similar vein as the weak Lindelöf property (see [3]).

Theorem 12. Let $X$ be a Hausdorff cellular-Lindelöf first-countable space. Then $c(X) \leq \mathfrak{c}$. 
Proof. Let $\mathcal{U}$ be a cellular family. Since $X$ is cellular-Lindelöf we can find a Lindelöf space $L \subset X$ such that $L \cap U \neq \emptyset$, for every $U \in \mathcal{U}$. But every Lindelöf first-countable space has cardinality at most the continuum, so $|L| \leq \mathfrak{c}$ and hence $|\mathcal{U}| \leq \mathfrak{c}$.

Corollary 13. Every Hausdorff cellular-Lindelöf first countable space has cardinality at most $2^{\mathfrak{c}}$.

Proof. This is an immediate consequence of the Hajnal-Juhász inequality $|X| \leq 2^{\chi(X) \cdot c(X)}$ (see, for example, [8], $2.15 \mathrm{~b}$ )) and Theorem 12.

Example 14. There are cellular-Lindelöf non-linearly Lindelöf Tychonoff spaces in $Z F C$.

Proof. Let $X=\Sigma\left(2^{\kappa}\right)=\left\{x \in 2^{\kappa}:\left|x^{-1}(1)\right| \leq \aleph_{0}\right\}$ with the topology induced from the usual product topology on $2^{\kappa}$. Then $X$ is ccc and hence it's cellular Lindelöf. Moreover $X$ is countably compact, so it can't be linearly Lindelöf, or otherwise it would be compact.

The above example should be contrasted with the fact that no example of a regular almost discretely Lindelöf non-Lindelöf space is known in ZFC. Also it seems that not even a consistent $T_{1}$ example of a discretely Lindelöf non-Lindelöf space is known at the moment.

Recall that a space is weakly Lindelöf if every open cover has a countable subcollection with a dense union (see [3]).

Example 15. There is a weakly Lindelöf $T_{2}$ non-cellular Lindelöf space.

Proof. This is Example 2.3 from [3]. Let $\kappa$ be a cardinal larger than the continuum and let $A$ be a countable dense subset of the irrationals. Define a topology on $X=(\mathbb{Q} \times \kappa) \cup A$ by declaring a basic neighbourhood of a point $(x, \alpha)$, where $x \in \mathbb{Q}$ and $\alpha<\kappa$, to be $(U \cap \mathbb{Q}) \times\{\alpha\}$, where $U$ is an open Euclidean interval containing $x$ and a basic neighbourhood of a point $y \in A$ to be of the form $(U \cap A) \cup((U \cap \mathbb{Q}) \times \kappa)$. This space is weakly Lindelöf because every open set containing $A$ is dense in $X$ (see [3]). However, it is not cellular-Lindelöf because it is first-countable but $c(X)>\mathfrak{c}$.

Note that Example 15 is only Hausdorff.

Question 2. Is there a Tychonoff example of a weakly Lindelöf noncellular Lindelöf space?

Question 3. Is there a cellular-Lindelöf non-weakly Lindelöf space?

Question 4. Is it true that every first-countable cellular-Lindelöf Hausdorff space has cardinality at most continuum? 
We conclude with a few more applications of the notion of $\theta$-free sequence. But first of all, let us note how the inequality $F_{\theta}(X)<F(X)$ can occur even for Urysohn spaces (that is spaces where each pair of distinct points can be separated by open neighbourhoods with disjoint closures).

Example 16. A Urysohn space $X$ such that $F_{\theta}(X)<F(X)$.

Proof. Let $X=K(\omega)$ be the Katětov extension of $\omega$. Recall that the underlying set of $K(\omega)$ is the same as the Cech-Stone compactification of the integers $\beta \omega$, that is, the set of all ultrafilters on $\omega$ (principal ultrafilters are identified with points of $\omega$ in the obvious way) but a local base at $p \in K(\omega)$ in the Katětov extension is given by $\{\{p\} \cup A: A \in p\}$. Note that $K(\omega) \backslash \omega$ is a closed discrete set of cardinality $2^{\mathfrak{c}}$. Therefore, $F(K(\omega))=2^{\mathfrak{c}}$.

The topology of $K(\omega)$ is finer than the topology of $\beta \omega$, however, for every $p \in K(\omega) \backslash \omega$, the topologies induced on $\{p\} \cup \omega$ by $K(\omega)$ and $\beta \omega$ are the same. Combining this with the observation that $\omega$ is dense in $K(\omega)$ we see that, for every open set $U \subset K(\omega)$, we have $\bar{U}=C l_{K(\omega)}(U)=C l_{K(\omega)}(U \cap \omega)=C l_{\beta \omega}(U \cap \omega)$. Therefore the closure of an open set in $K(\omega)$ is actually a clopen set in $\beta \omega$.

Let $S=\left\{x_{\alpha}: \alpha<\kappa\right\}$ be a $\theta$-free sequence in $K(\omega)$. Fix $\alpha<\kappa$. For every $\gamma \in \kappa \backslash \alpha$ there exists an open neighbourhood $U_{\gamma}$ of $x_{\gamma}$ such that $\overline{U_{\gamma}} \cap\left\{x_{\beta}: \beta<\alpha\right\}=\emptyset$. Since the set $\bigcup\left\{\overline{U_{\gamma}}: \gamma \in \kappa \backslash \alpha\right\}$ is open in $\beta \omega$, we see that $S$ is a left-separated set in $\beta \omega$. Thus, we have that $|S| \leq \mathfrak{c}$ and hence $F_{\theta}(K(\omega)) \leq \mathfrak{c}$.

Given a space $X$, a set $A \subseteq X$ is $\theta$-dense in $X$ if $[A]_{\theta}=X$. The $\theta$-density $\theta d(X)$ is the smallest cardinality of a $\theta$-dense subset of $X$.

Theorem 17. Let $X$ be a space. If a cardinal $\lambda$ satisfying $F_{\theta}(X)<$ $\lambda \leq\left(2^{F_{\theta}(X)}\right)^{+}$is a caliber of $X$, then $\theta d(X) \leq 2^{F_{\theta}(X)}$.

Proof. Let $F_{\theta}(X)=\kappa$ and assume by contradiction that the $\theta$-density of $X$ is bigger than $2^{\kappa}$. Fix a choice function $\eta: \mathcal{P}(X) \backslash\{\emptyset\} \rightarrow X$. We will define by induction an increasing family $\left\{A_{\alpha}: \alpha<\lambda\right\}$ of subsets of $X$ of cardinality not exceeding $2^{\kappa}$ and a family $\left\{U_{\alpha}: \alpha<\lambda\right\}$ of non-empty open subsets of $X$ in such a way that:

(1) $\left[A_{\alpha}\right]_{\theta} \cap \overline{U_{\alpha}}=\emptyset$;

(2) if $\mathcal{V} \subseteq\left\{U_{\beta}: \beta<\alpha\right\}$ satisfies $|\mathcal{V}| \leq \kappa$ and $\bigcap \mathcal{V} \neq \emptyset$, then $\eta(\bigcap \mathcal{V}) \in A_{\alpha}$

To justify the inductive construction, let us assume to have already defined the sets $\left\{A_{\beta}: \beta<\alpha\right\}$ and $\left\{U_{\beta}: \beta<\alpha\right\}$. Since $\alpha<\lambda$ and $\lambda \leq\left(2^{F_{\theta}(X)}\right)^{+}$, we have $\left|\left\{U_{\beta}: \beta<\alpha\right\}\right| \leq|\alpha| \leq 2^{\kappa}$. Consequently, 
the set $B=\left\{\eta(\bigcap \mathcal{V}): \mathcal{V} \subseteq\left\{U_{\beta}: \beta<\alpha\right\},|\mathcal{V}| \leq \kappa\right.$ and $\left.\bigcap \mathcal{V} \neq \emptyset\right\}$ has cardinality not exceeding $2^{\kappa}$. Then, let $A_{\alpha}=B \cup \cup\left\{A_{\beta}: \beta<\alpha\right\}$. As we are assuming that the $\theta$-density of $X$ is bigger than $2^{\kappa}$, we may find a non-empty open set $U_{\alpha}$ such that $\left[A_{\alpha}\right]_{\theta} \cap \overline{U_{\alpha}}=\emptyset$.

Since $\lambda$ is a caliber of $X$, there exists a set $S \subseteq \lambda$ such that $|S|=\lambda$ and $\bigcap\left\{U_{\alpha}: \alpha \in S\right\} \neq \emptyset$. We may fix an increasing mapping $f: \lambda \rightarrow S$. Observe now that we are assuming $\kappa^{+} \leq \lambda$. For any $\alpha<\kappa^{+}$let $x_{\alpha}=$ $\eta\left(\bigcap\left\{U_{f(\xi)}: \xi \leq \alpha\right\}\right)$. We claim that the set $\left\{x_{\alpha}: \alpha<\kappa^{+}\right\}$so obtained is a $\theta$-free sequence in $X$. To check this, fix $\alpha<\kappa^{+}$and observe that for each $\beta<\alpha$ we have $x_{\beta} \in A_{f(\beta)+1} \subseteq A_{f(\alpha)}$. Moreover, for each $\beta \geq \alpha$ the set $U_{f(\alpha)}$ occurs in the definition of $x_{\beta}$ and consequently $x_{\beta} \in U_{f(\alpha)}$. This means that $\left\{x_{\beta}: \beta<\alpha\right\} \subseteq A_{f(\alpha)}$ and $\left\{x_{\beta}: \alpha \leq \beta<\kappa^{+}\right\} \subseteq U_{f(\alpha)}$. Therefore $\left[\left\{x_{\beta}: \beta<\alpha\right\}\right]_{\theta} \cap \overline{\left\{x_{\beta}: \alpha \leq \beta<\kappa^{+}\right\}} \subseteq\left[A_{f(\alpha)}\right]_{\theta} \cap \overline{U_{f(\alpha)}}=\emptyset$. The validity of the claim contradicts the hypothesis and the proof is then complete.

Corollary 18. Let $X$ be a regular space. If a cardinal $\lambda$ satisfying $F(X)<\lambda \leq\left(2^{F(X)}\right)^{+}$is a caliber of $X$, then $d(X) \leq 2^{F(X)}$

Corollary 19. Let $X$ be a regular sequential space with no uncountable free sequence and $\lambda \leq \mathfrak{c}^{+}$be an uncountable cardinal such that $\lambda$ is a caliber of $X$. Then $|X| \leq \mathfrak{c}$.

\section{ACKNowledgements}

The first-named author was partially supported by a grant of the Group GNSAGA of INDAM. The second-named author is grateful to FAPESP for financial support through postdoctoral grant 2013/146401, Discrete sets and cardinal invariants in set-theoretic topology. Part of the research for the paper was carried out when he visited the firstnamed author at the University of Catania in December 2016. He thanks his colleagues there for the warm hospitality. The authors are grateful to Lajos Soukup for spotting an error in an earlier version of the paper.

\section{REFERENCES}

[1] A. Arhangel'skii, On the cardinality of bicompacta satisfying the first axiom of countability, Soviet Math. Dokl. 10 (1969), 951-955.

[2] Z. Balogh, On density and the number of $G_{\delta}$ points in somewhat Lindelöf spaces, Topology Proc. 27 (2003), 9-14.

[3] M. Bell, J. Ginsburg and G. Woods, Cardinal inequalities for topological spaces involving the weak Lindelöf number, Pacific Journal of Mathematics 79 (1978), $37-45$. 
[4] A. Bella and F. Cammaroto, On the cardinality of Urysohn spaces, Canad. Math. Bull. 31 (1988), 153-158.

[5] A. Dow, An introduction to applications of elementary submodels to topology, Topology Proc. 13 (1988), no. 1, 17-72.

[6] R. Engelking, General Topology, PWN, Warsaw, 1977.

[7] R. E. Hodel, Arhangelskii's solution to Alexandroff's problem, Topology Appl. 153 (2006), 2199-2217.

[8] I. Juhász, Cardinal Functions in Topology - Ten Years Later, Math. Centre Tracts 123, 1980, Amsterdam.

[9] I. Juhász, L. Soukup and Z. Szentmiklóssy, First-countable and almost discretely Lindelöf $T_{3}$ spaces have cardinality at most continuum, preprint, arXiv:1612.06651.

[10] I. Juhász, V.V. Tkachuk and R.G. Wilson, Weakly linearly Lindelöf monotonically normal spaces are Lindelöf, preprint, arXiv:1610.04506.

[11] K. Kunen, Set Theory, Studies in Logic, n. 34, College Publications, London, 2011.

[12] N. Veličko, H-closed topological spaces, Math. Sb. (NS) 70 (1966), pp 98-112; Amer. Math. Soc. Transl. 78 (Ser. 2) (1969), pp-103-118.

Department of Mathematics and Computer Science, University of Catania, Cittá universitaria, viale A. Doria 6, 95125 Catania, Italy

E-mail address: bella@dmi.unict.it

Instituto de Matematica e Estatistica (IME-USP), Universidade De Sao Paulo, Rua do Matao, 1010 - Cidade Universitaria, 05508-090 SaO PAULO - SP, BRAZIL

Current address: Department of Mathematics and Computer Science, University of Catania, Cittá universitaria, viale A. Doria 6, 95125 Catania, Italy

E-mail address: santidspadaro@gmail.com 